\title{
PRACTICAL FINITE ELEMENT PROCEDURE FOR ACHIEVING MESH OBJECTIVITY IN LOCAL BUCKLING ANALYSIS OF STEEL STRUCTURES BY BEAM ELEMENTS
}

\author{
Eiki Yamaguchi \\ Professor, Department of Civil Engineering, \\ Kyushu Institute of Technology, Tobata, Kitakyushu, Japan \\ E-mail: yamaguch@civil.kyutech.ac.jp
}

Received: 31 May 2007; Revised: 24 March 2008; Accepted: 27 March 2008

\begin{abstract}
Since the nonlinear finite element analysis of a steel structure by shell/solid elements is expensive, effort has been made to conduct the local buckling analysis of a steel structure by beam elements. To this end, the structural deterioration due to local buckling of a steel member is implemented in constitutive relationship. The approach inevitably leads to the constitutive relationship of softening type, which however does not readily yield mesh objective result. The present study proposes a finite element procedure to overcome the problem: in a local-buckling zone, average state variables instead of local state variables are used. The effectiveness of the proposed procedure is verified by solving example problems. Moreover, the applicability of a simple trilinear type of constitutive relationship associated with the proposed beam-element analysis is investigated in comparison with shell-element analysis by ABAQUS.
\end{abstract}

Keywords: Steel structure; local buckling; beam element; mesh objective; softening-type constitutive relationship

\section{INTRODUCTION}

Structural steel has been used exclusively as a thin-walled member in its civil and building application. One of its major failure modes is a local buckling, which may deteriorate the member strength considerably. Therefore, the phenomenon has been studied for many years.

Nevertheless, a number of steel structures underwent local buckling in the 1995 Hyogo-ken Nambu Earthquake also known as Kobe Earthquake, which was the very first time steel structures in service were damaged so badly in Japan, surprising many Japanese researchers who had confidence in the safety of steel structures. Quite a few experimental researches were then conducted in Japan [1]. Analytical approach was also taken and the local buckling has been simulated successfully not only by experiment but also by 3-dimensional finite element analysis using shell/solid elements [2-3].

Along this line, effort has been made also to analyze local-buckling behavior by beam elements for the reduction of computational cost. To this end, the structural deterioration due to local buckling is implemented in constitutive relationship, which is inevitably of a softening type [4-6]. In fact, the reduction of computational cost has been a challenging subject in various engineering fields. For buckling analysis, for example, the work of Little is noted [7].

The softening type of constitutive relationship has been used for the cracking analysis of concrete. A problem associated with this approach is dependence on finite element mesh: numerical result by the simple application of a softening-type constitutive relationship would not converge as the mesh becomes finer. In other words, mesh objective result is not obtained. To solve this problem, nonlinear fracture mechanics has been explored and, to be specific, it has been solved by controlling the slope of a softening branch of a constitutive relationship [8-10]. However, the issue of mesh objectivity has not been thoroughly understood in conjunction with the local buckling of steel structures. 


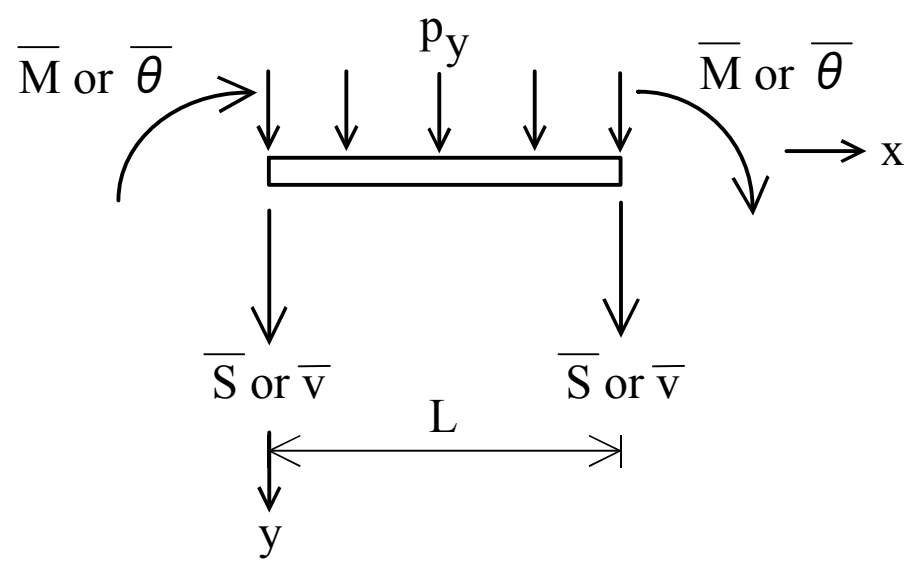

Figure 1. Bending Problem of Beam

In the present study, the mesh objectivity is first looked into, which shows that just like in the concrete cracking analysis, result does not converge with the refinement of mesh in the analysis of a steel structure by beam elements coupled with the simple application of the softening-type constitutive relationship. The objective of the present study is then to propose a finite element procedure to yield mesh objective result. The effectiveness of the proposed procedure is demonstrated by solving example problems. Nonlinear analysis of a two-dimensional beam is conducted to this end.

\section{CONVENTIONAL FORMULATION FOR NONLINEAR BEAMS}

The conventional finite element procedure for the bending behavior of a two-dimensional beam is briefly described in this section for the sake of self-sufficiency. Nonlinearity is in constitutive relationship.

Assuming that displacement is small, the Bernoulli-Euler beam theory for the beam shown in Figure 1 leads to the following governing equations:

Equilibrium equations:

$$
M^{\prime \prime}-p_{y}=0
$$

Curvature-displacement relationship: $\quad \kappa=v^{\prime \prime}$

Moment-curvature relationship:

$$
\dot{M}=C \dot{\kappa}
$$

Boundary conditions: $\bar{S}=-S$ or $\bar{v}=v, \bar{M}=-M$ or $\bar{\theta}=v^{\prime}$ at $x=0$

$$
\bar{S}=S \text { or } \bar{v}=v, \quad \bar{M}=M \text { or } \bar{\theta}=v^{\prime} \quad \text { at } x=L
$$

where $M, p_{y}, \kappa, v, S$ and $\theta$ are bending moment, distributed load in the $y$-direction, curvature, displacement in the $y$-direction, shear force and rotational angle, respectively. A quantity with the top bar such as $\bar{S}$ is the prescribed one. The top dot such as that in $\dot{M}$ and the prime such as that in $M^{\prime}$ represent the rate and the derivative with respect to $x$, respectively. $C$ is bending rigidity and is equal to EI (the product of the Young Modulus and the moment of inertia) at the initial stage of deformation. As deformation increases, $C$ may vary, initiating nonlinear behavior. 


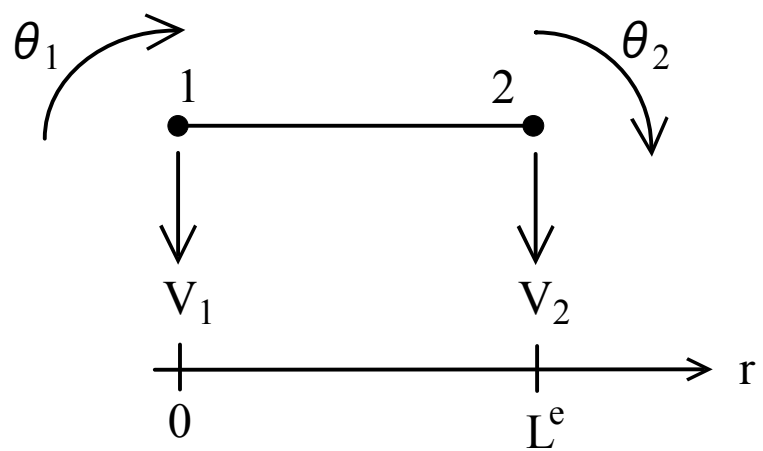

Figure 2. Standard 2-node Four-d.o.f. Beam Element

The application of the weighted residual method to these equations yields

$$
H=\int_{0}^{L} M w^{\prime \prime} d x-\int_{0}^{L} p_{y} w d x-\left[\bar{S} w+\bar{M} w^{\prime}\right]_{x=0, L}=0
$$

where $w$ is the weight function. Using a standard 2-node four-d.o.f. beam element shown in Figure 2 [11] and the Galerkin method, the displacement and the weight are discretized as

$$
\begin{aligned}
& v=\mathbf{N U}^{e}=\left[\begin{array}{llll}
N_{V 1} & N_{\theta 1} & N_{V 2} & N_{\theta 2}
\end{array}\right]\left[\begin{array}{llll}
V_{1} & \theta_{1} & V_{2} & \theta_{2}
\end{array}\right]^{T} \\
& w=\mathbf{N} \mathbf{W}^{e}=\left[\begin{array}{llllll}
N_{V 1} & N_{\theta 1} & N_{V 2} & N_{\theta 2}
\end{array}\right]\left[\begin{array}{lllll}
W_{V 1} & W_{\theta 1} & W_{V 2} & W_{\theta 2}
\end{array}\right]^{T}
\end{aligned}
$$

where

$$
N_{V 1}=1-\frac{3 r^{2}}{L^{e 2}}+\frac{2 r^{3}}{L^{e 3}}, \quad N_{\theta 1}=r-\frac{2 r^{2}}{L^{e}}+\frac{r^{3}}{L^{e 2}}, \quad N_{V 2}=\frac{3 r^{2}}{L^{e 2}}-\frac{2 r^{3}}{L^{e 3}}, \quad N_{\theta 2}=-\frac{r^{2}}{L^{e}}+\frac{r^{3}}{L^{e 2}}
$$

$L^{e}$ is the length of a beam element and $r$ is the local coordinate attached to each element, taking 0 at Node 1 and $L^{e}$ at Node 2 .

The discretization by this beam element gives the following expression for each element:

$H^{e}=\mathbf{W}^{e T}\left(\mathbf{F}_{\mathrm{IN}}^{e}-\mathbf{F}_{\mathrm{EX}}^{e}\right)$

where

$$
\begin{aligned}
& \mathbf{F}_{\mathrm{IN}}^{e}=\int_{L^{e}} \mathbf{B} M d r \\
& \mathbf{F}_{\mathrm{EX}}{ }^{e}=\int_{L^{e}} \mathbf{N} p_{y} d r+[\bar{S}+\bar{M}]_{r=0, L^{e}} \\
& \mathbf{B}=\frac{d^{2} \mathbf{N}}{d r^{2}}=\left[-\frac{6}{L^{e 2}}+\frac{12 r}{L^{e 3}}-\frac{4}{L^{e}}+\frac{6 r}{L^{e 2}} \frac{6}{L^{e 2}}-\frac{12 r}{L^{e 3}}-\frac{2}{L^{e}}+\frac{6 r}{L^{e 2}}\right]
\end{aligned}
$$


The assemblage of all the element contributions and the arbitrariness of the weight function yield the following discretized governing equations:

$$
\mathbf{F}_{\mathrm{IN}}-\mathbf{F}_{\mathrm{EX}}=\mathbf{0}
$$

Because of the nonlinearity of Eq. 3, Eq. 14 is a set of nonlinear equations. To obtain the solution, Eq. 14 is linearized as

$$
\mathbf{K}\left(\mathbf{U}^{(m)}\right) \Delta \mathbf{U}^{(m)}=\mathbf{F}_{\mathrm{EX}}-\mathbf{F}_{\mathrm{IN}}\left(\mathbf{U}^{(m)}\right)
$$

The coefficient matrix $\mathbf{K}$, also known as the stiffness matrix, has the following expression at an element level:

$$
\mathbf{K}^{e}=\int_{L^{e}} \mathbf{B}^{T} C \mathbf{B} d r=\frac{C}{L^{e 3}}\left[\begin{array}{cccc}
12 & 6 L^{e} & -12 & 6 L^{e} \\
& 4 L^{e 2} & -6 L^{e} & 2 L^{e 2} \\
& & 12 & -6 L^{e} \\
s y m . & & & 4 L^{e 2}
\end{array}\right]
$$

Eq. 15 is solved for $\Delta \mathbf{U}^{(m)}$ and $\mathbf{U}$ is updated. The procedure is repeated until convergence is obtained. The superscript $m$ in the parenthesis shows the number of iteration.

In reality, shear deformation contributes to deflection. The Timoshenko beam theory can be used to include the effect of shear deformation. In this theory, the deflection is expressed as the sum of those due to bending and shear deformation:

$$
\begin{aligned}
& V_{1}=V_{1}^{b}+V_{1}^{s} \\
& V_{2}=V_{2}^{b}+\left(V_{1}^{s}-\frac{S L^{e}}{G k A}\right)
\end{aligned}
$$

where the superscripts $b$ and $s$ stand for the contributions due to bending and shear deformation, respectively, $G$ is the shear modulus, $A$ is a cross-sectional area and $k$ is the correction factor that accounts for the difference from actual shear stress distribution over a cross section. The value of $k$ depends on the cross section of a beam, and for a thin-walled member the ratio of the cross-sectional area of a web over the whole cross-sectional area $A$ can be assigned to $k$ [12]. The coefficient matrix $\mathbf{K}^{e}$ for the Timoshenko beam theory is then given by

$$
\mathbf{K}^{e}=\frac{C}{L^{e 3}(1+\phi)}\left[\begin{array}{cccc}
12 & 6 L^{e} & -12 & 6 L^{e} \\
& (4+\phi) L^{e 2} & -6 L^{e} & (2-\phi) L^{e 2} \\
& & 12 & -6 L^{e} \\
s y m . & & & (4+\phi) L^{e 2}
\end{array}\right]
$$

where 


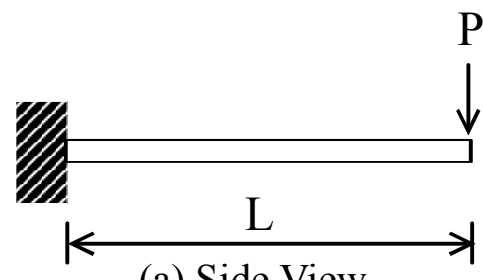

(a) Side View

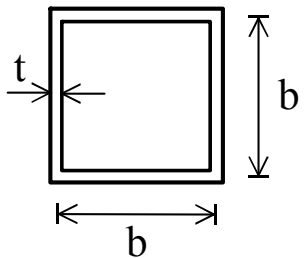

(b) Cross Section

Figure 3. Cantilever Beam

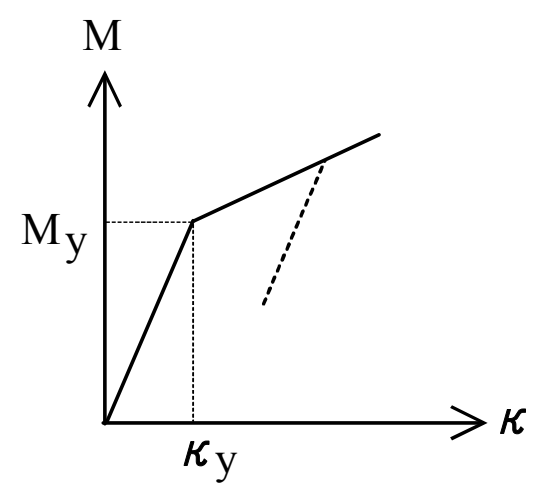

(a) Type I

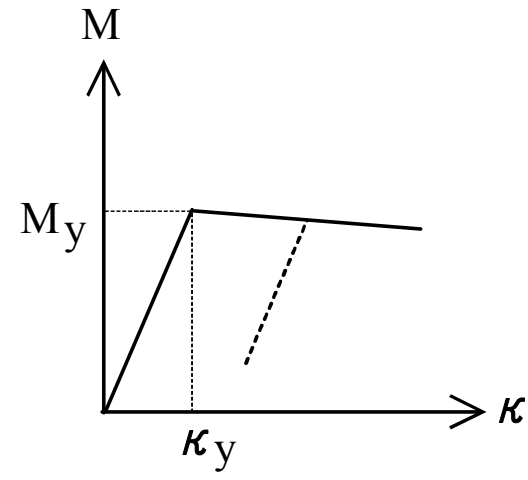

(b) Type II

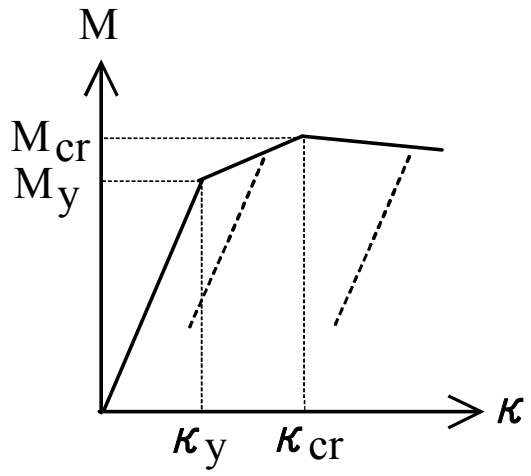

(c) Type III

Figure 4. Bending Moment $M$-curvature $\kappa$ Relationships

$\phi=\frac{12 C}{G k A L^{e 2}}$

\section{NUMERICAL ANALYSIS I: MESH DEPENDENCE}

The two-dimensional behavior of a steel cantilever beam subjected to a concentrated load at the free end (Figure 3) is employed as a numerical example herein. The beam length $L$ is $10 \mathrm{~m}$ and the cross section is a square box consisting of four thin plates, whose width $b$ is $2000 \mathrm{~mm}$ and thickness $t$ is $40 \mathrm{~mm}$. The Young Modulus $E$ is $206 \mathrm{kN} / \mathrm{mm}^{2}$ and the yield stress $\sigma_{y}$ is 235 $\mathrm{N} / \mathrm{mm}^{2}$. The main objective of this section is to shed light on the difficulty in dealing with the constitutive model of a softening type. To this end, it is sufficient to consider only the bending behavior of the beam: no shear deformation is taken into account in this section.

For nonlinear behavior, three simple constitutive relationships between the bending moment $M$ and the curvature $\kappa$ shown in Figure 4 are assumed. Type I represents a typical elasto-plastic behavior. No descending branch (softening branch) exists. Type II has no hardening branch but has the softening branch, which embodies the deterioration due to local buckling without undergoing plastic deformation. Type III includes both hardening and softening branches: the elastic behavior is followed by the elasto-plastic behavior, which then leads to the deterioration due to local buckling. The slopes of the hardening and softening branches are assumed to be $E I / 10$ and $-E I / 100$, respectively. For Type III, $\kappa_{c r}$ is assumed to be twice as large as $\kappa_{y}$, i.e. $\kappa_{c r}=2 \kappa_{y}$. In the hardening and softening branches, unloading behavior may take place. The slope of the unloading $M-\kappa$ relationship is the same as that of the elastic behavior, which is illustrated by dotted lines in Figure 4. 


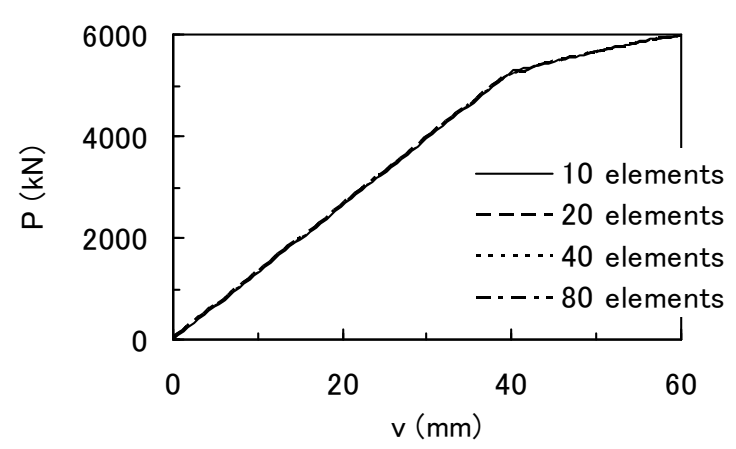

(a) Type I

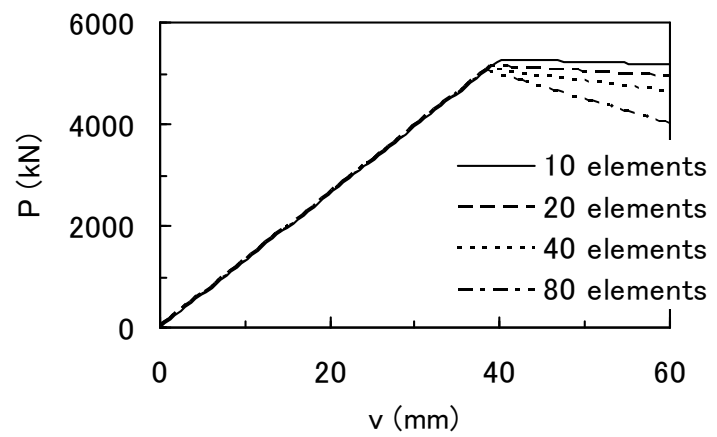

(b) Type II

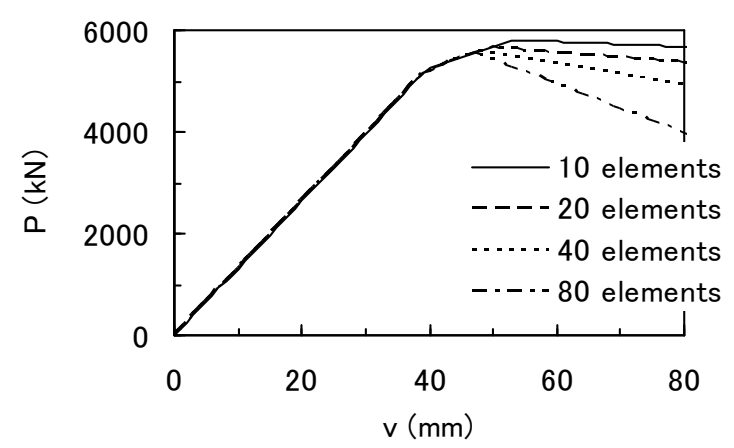

(c) Type III

Figure 5. Load $P$ - Displacement $v$ Curves of Cantilever Beam

Using the beam elements described earlier, four finite element meshes are constructed to see the influence of the mesh on numerical result. The four meshes use 10,20, 40 and 80 elements, respectively. All the elements in each mesh are equal in length.

The numerical results of Type I are presented in the form of the load $P$ - displacement $v$ relationship at the loading point in Figure 5(a). Even though dependence on the mesh is not significant, closer observation reveals the tendency of the convergence: as the element length becomes smaller, the result tends to converge. The 20-, 40- and 80-element meshes yield practically the same response. For instance, at $v=60 \mathrm{~mm}, P$ is equal to $5993 \mathrm{kN}$ in the case of the 10 -element mesh while it is just about $5970 \mathrm{kN}$ in the other three cases. Mesh objective result is thus obtained with Type I.

Figure 5(b) shows the results of Type II. The dependence on the mesh is recognized more clearly, as the results by the four meshes are very different from each other. Unlike the previous result of Type I, no tendency of the convergence is observed in this type of constitutive model, which indicates that the result herein is not mesh objective.

The $P-v$ relationship due to Type III is presented in Figure 5(c). This figure clearly indicates the dependence on the mesh. As the mesh becomes finer, the dependence reduces in the hardening portion of the $P-v$ relationship while such tendency is not observed in the softening portion. The problem associated with the softening type of constitutive relationship is made apparent again by Type III. 


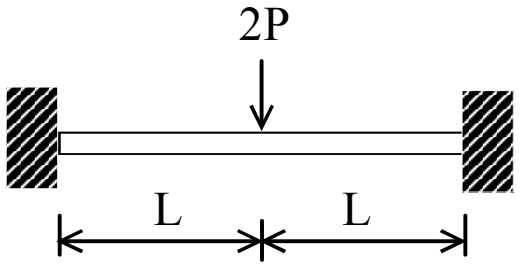

(a) Whole Beam

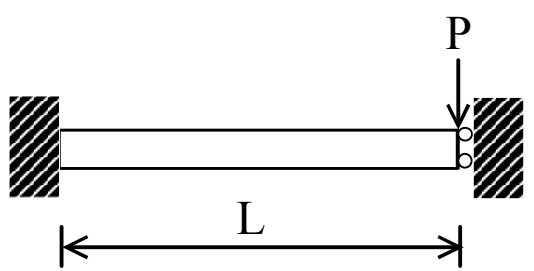

(b) Half Beam

Figure 6. Fixed-fixed Beam

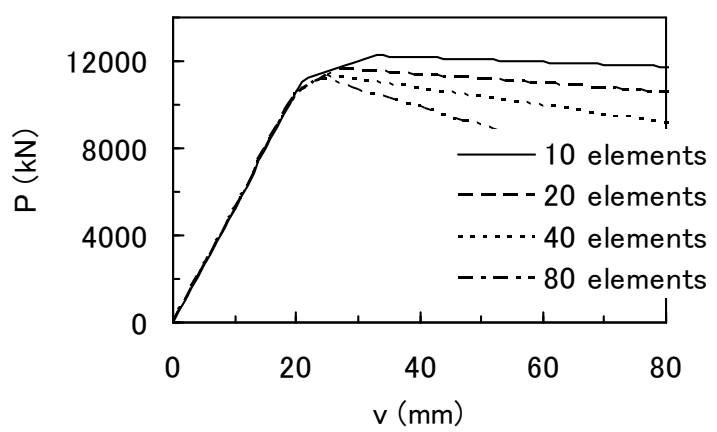

Figure 7. Load $P$ - Displacement $v$ Curves of Fixed-fixed Beam (Type III)

Additionally, a statically indeterminate structure of a steel fixed-fixed beam with a concentrated load at the mid-span (Figure 6(a)) is also analyzed with Type III. The total length of the beam $2 L$ is $20 \mathrm{~m}$ and the cross section is the same as that of the cantilever (Figure 3(b)). Since it is sufficient to consider only a half of the beam due to symmetry, the analysis of the beam shown in Figure $6(\mathrm{~b})$ is conducted. The numerical results in the form of the $P-v$ relationship at the loading point are plotted in Figure 7. This figure shows that mesh objective result is not obtained in the statically indeterminate structure as well.

\section{PROPOSED FORMULATION FOR SOFTENING BEHAVIOR}

The following observations are generally made in the local buckling phenomenon of a steel thin-walled member:

1. Region subjected to local buckling is limited. (The region is hereafter called the local-buckling zone or LBZ for short.)

2. Strain distribution in LBZ depends on buckling mode and is very complicated in general, so that strain in LBZ varies from point to point wildly.

It is noteworthy that the study on LBZ has been conducted, providing the following empirical formula for evaluating the length of LBZ of a box-section member [13]:

$L_{L B Z}=\operatorname{Min}(0.7 b, a)$

where $L_{L B Z}$ is the length of LBZ, $a$ is the distance between two adjacent diaphragms and $b$ is the width of a flange. Min indicates that the smaller of the two values in the parenthesis shall be taken. 
Making use of the above observations, the constitutive relationship between $\dot{M}$ and $\dot{\kappa}$ in LBZ is assumed to be controlled by the average quantity in LBZ in the present study. To be specific, the constitutive relationship of Eq. 3 is replaced by

$\dot{M}=C(\kappa) \dot{\kappa} \quad$ for $\kappa_{a v} \leq \kappa_{c r}$

$\dot{M}=C\left(\kappa_{a v}\right) \dot{\kappa}$ for $\kappa_{a v}>\kappa_{c r}$

where $\kappa_{a v}$ is the average curvature in LBZ. Specifically, $\kappa_{a v}$ is defined by

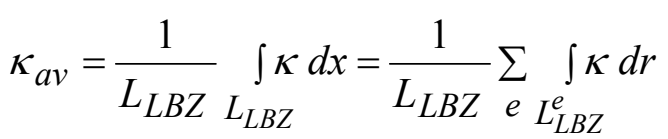

where $L_{L B Z}^{e}$ is the length of the element located in LBZ. The proposed approach may be viewed as a kind of nonlocal formulation with $L_{L B Z}$ being the characteristic length $[14,15]$.

For the beam element employed in this study, the curvature varies as follows:

$\kappa=\frac{d^{2} v}{d r^{2}}=\mathbf{B} \mathbf{U}^{e^{T}}$

As Eq. 13 indicates, $\mathbf{B}$ is a set of linear functions with respect to $r$. Therefore, $\kappa$ varies in a linear fashion within a beam element. Eq. 23 can then be rewritten as

$\kappa_{a v}=\frac{1}{L_{L B Z}} \sum_{e} L_{L B Z}^{e} \kappa\left(\frac{L_{L B Z}^{e}}{2}\right)$

where $\kappa\left(L_{L B Z}^{e} / 2\right)$ is the value of $\kappa$ evaluated at the middle of the element. If the lengths of the elements in LBZ are equal to each other, the above equation can be further simplified as

$\kappa_{a v}=\frac{1}{N} \sum_{e} \kappa\left(\frac{L_{L B Z}^{e}}{2}\right)$

where $N$ is the number of elements located in LBZ.

It is also noted that Eq. $22 \mathrm{~b}$ leads to

$\dot{M}_{a v}=\frac{1}{L_{L B Z}} \int_{L_{L B Z}} \dot{M} d r=\frac{1}{L_{L B Z}} \int_{L_{L B Z}} C\left(\kappa_{a v}\right) \dot{\kappa} d r=\frac{C\left(\kappa_{a v}\right)}{L_{L B Z}} \underset{L_{L B Z}}{\int} \dot{\kappa} d r=C\left(\kappa_{a v}\right) \dot{\kappa}_{a v}$

where $\dot{M}_{a \nu}$ is the average bending moment in LBZ. Since deformation in LBZ is complicated, experiments provide information only for this class of constitutive relationship in practice, i.e. the relationship only in terms of average state variables $[5,6]$. In other words, $C\left(\kappa_{a v}\right)$ can be determined experimentally. 
It is noted that the finite element procedure described earlier can be used with this constitutive relationship of Eq. 22.

\section{NUMERICAL ANALYSIS II: EFFECTIVENESS OF PROPOSED APPROACH}

The two problems of the cantilever beam (Figure 3) and a half of the fixed-fixed beam (Figure 6(b)) are solved by the proposed approach. Since the cross section at the fixed end does not deform and a diaphragm is placed at the cross section under the concentrated load, LBZ can develop near the fixed end in both beams and also near the loading point in the case of the fixed-fixed beam. Following Eq. 21, it is decided that the length of each LBZ is $1400 \mathrm{~mm}$.

The proposed constitutive relationship of Eq. 22 is applied to these LBZs. The values of the parameters in the constitutive relationship are assumed to be the same as those employed in the previous analysis, i.e. Numerical analysis I. However, the physical meaning of the critical value $\kappa_{c r}$ for the judgment on the initiation of local buckling has become different: it is now in terms of the average curvature $\kappa_{a v}$ while it is local curvature $\kappa$ in the previous analysis. Outside LBZ no local buckling takes place so that no structural deterioration occurs. The constitutive relationship outside LBZ is therefore assumed to be given by Type I (Figure 4(a)) and the parameters take the same values as those employed previously.

Four finite element meshes are employed in this analysis. In the coarsest mesh, seven elements are used for the whole beam: LBZ is modeled by one element and the remaining zone is modeled by the rest of the elements. The element mesh is refined by halving the element length, so the four meshes consist of 7, 14, 28 and 56 elements, respectively, and LBZs in the four meshes are modeled by 1, 2, 4 and 8 elements, respectively. The elements in LBZ are equal in length and the elements in the remaining zone are equal in length as well, but the element lengths in LBZ and the remaining zone are slightly different from each other.

The results are shown in Figures 8 and 9. Both figures indicate that the variations due to the difference in mesh are much smaller than the counterparts in the previous analysis with Type III (Figures 5(c) and 7). Not only have the variations been reduced but the convergence is observed as the mesh becomes smaller, which can be realized more clearly in Figures $8(\mathrm{~b})$ and $9(\mathrm{~b})$. The convergence appears to be relatively slow in the fixed-fixed beam, yet the 28- and 56-element meshes have yielded indistinguishable responses. The effectiveness of the proposed approach is thus verified.

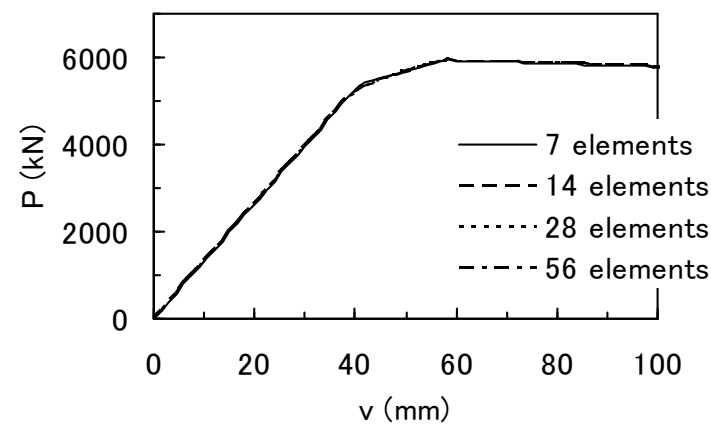

(a) Overall Range

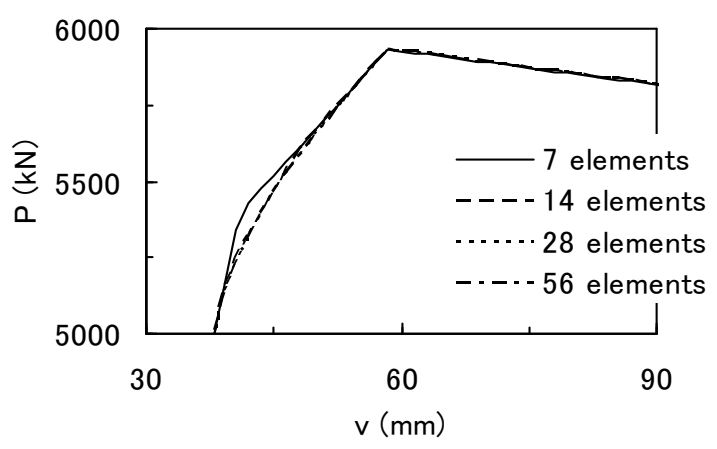

(b) Close-up of Peak Region

Figure 8. Load $P$ - Displacement $v$ Curves of Cantilever Beam by Proposed Approach 


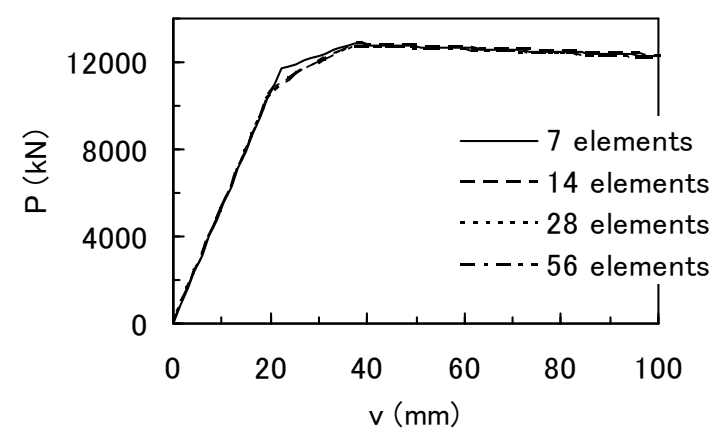

(a) Overall Range

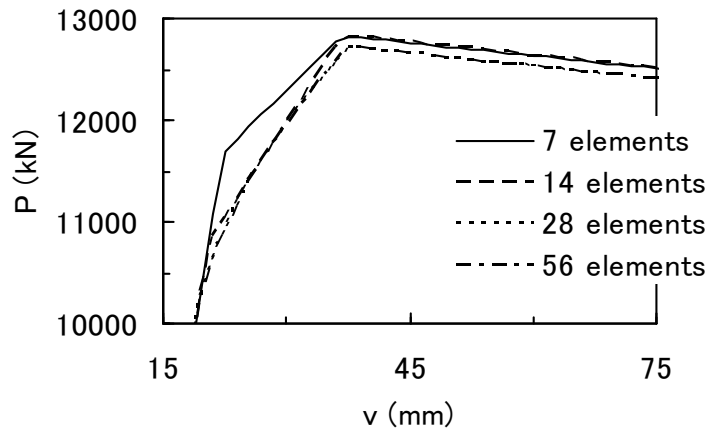

(b) Close-up of Peak Region

Figure 9. Load $P$ - Displacement $v$ Curves of Fixed-fixed Beam by Proposed Approach

\section{NUMERICAL ANALYSIS III: APPLICABILITY OF TRILINEAR TYPE OF CONSTITUTIVE RELATIONSHIP IN TERMS OF BENDING MOMENT $M$ AND CURVATURE $\kappa$}

The finite element procedure for achieving mesh objectivity is proposed and its effectiveness is confirmed in the above. Since the objective of the present study is to propose the mesh-objective finite element procedure, the constitutive relationship used is one of the simplest softening-type models. Yet it may be interesting to examine the applicability of this simple trilinear type of constitutive relationship from practical point of view. Therefore, the comparative study between the proposed beam-element analysis with the trilinear type of constitutive relationship and the shell-element analysis by ABAQUS [16] is carried out herein.

The ABAQUS analysis takes advantage of symmetry, and each beam is modeled by 4040 four-node shell elements. The material in the ABAQUS analysis is assumed to be a von Mises type of elasto-plastic material and its uniaxial behavior is bilinear with the second slope being equal to $E / 100$. The Poisson ratio is 0.3 , and the Young Modulus $E$ and the yield stress $\sigma_{y}$ take the same values as those in the previous beam-element analysis.

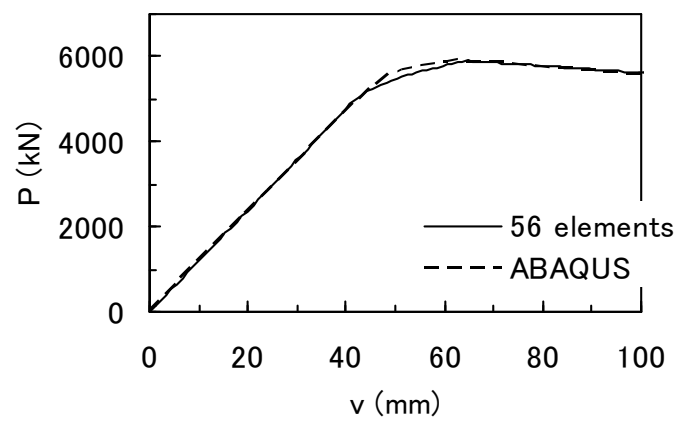

(a) Load $P$ - Displacement $v$ Curves

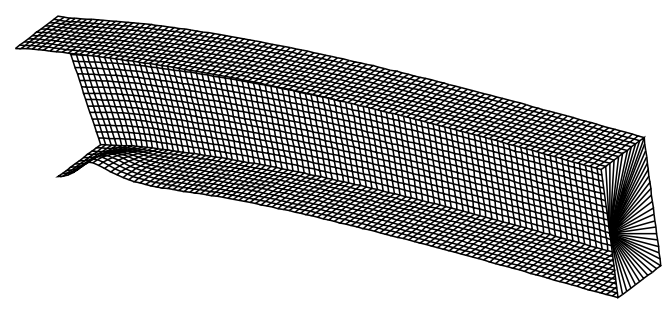

(b) Deformed Configuration (ABAQUS)

Figure 10. Analysis of Cantilever Beam under Concentrated Load

Since in the ABAQUS analysis, shear deformation contributes to deflection, the Timoshenko beam theory needs to be incorporated into the proposed beam-element analysis. This can be done simply by employing Eq. 19 instead of Eq. 16. Furthermore, care must be taken in the determination of the values of the bending rigidities $C$ and the critical curvature $\kappa_{c r}$, since they are not pure material properties but include the structural characteristics, particularly cross-sectional properties. To this 
end, the cantilever problem with the concentrated load (Figure 3) is solved first by ABAQUS. Referring to this ABAQUS result, the bending rigidities and the critical curvature are evaluated by trial and error, and $C=E I / 11.20$ for the hardening behavior, $C=-E I / 46.82$ for the softening behavior and $\kappa_{c r}=2.07 \kappa_{y}$ are obtained. These values are to be used not only for the cantilever problem with the concentrated load, but also for all the other problems.

The same four meshes as those described in the previous section are used again in this comparative study. As is in the previous examples (Figures 8 and 9), the numerical result converges also herein with the refinement of the mesh: the responses obtained by the 28-element mesh and the 56 -element mesh are practically the same. Because of the convergence, only the numerical result by the 56-element mesh is presented in Figure 10(a) together with that due to ABAQUS. The difference in the maximum load between the two results is $0.10 \%$. The post-peak deformed configuration obtained by the ABAQUS analysis is given in Figure 10(b). For the clear presentation, the deformation is magnified by the factor of 30 in this figure. The local buckling is clearly observed in the compression flange near the fixed end. Compared to this ABAQUS result, the LBZ length of $1400 \mathrm{~mm}$ employed in the proposed beam-element analysis appears justifiable.

Replacing the concentrated load with uniformly distributed load, the cantilever beam (Figure 11(a)) is analyzed again. All the values of the parameters in the constitutive relationship of the proposed approach including the values of $C$ and $\kappa_{c r}$ remain the same as those in the cantilever-beam problem with the concentrated load. The numerical result obtained is presented in Figure 11(b) together with that due to ABAQUS. Because of the convergence, only the numerical result by the 56-element mesh is given. The two results are in good agreement with each other: the difference in the maximum load is $3.56 \%$. The deformed configuration in the ABAQUS analysis is presented in Figure 11(c). The deformation is magnified by the factor of 30. The local buckling is clearly observed in the compression flange near the fixed end, and the LBZ length of $1400 \mathrm{~mm}$ appears good also in this problem.

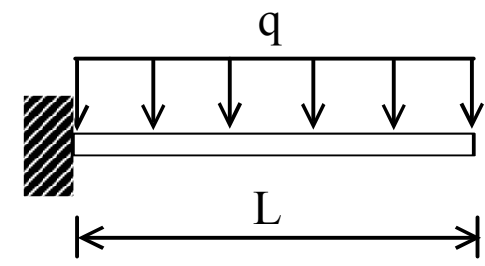

(a) Cantilever Beam under Distributed Load

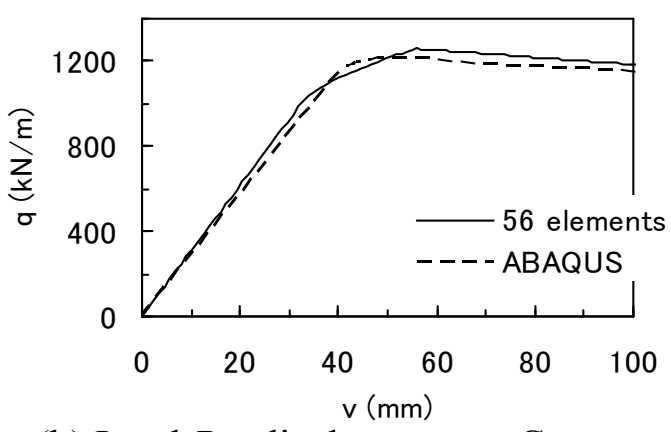

(b) Load $P$-displacement $v$ Curves

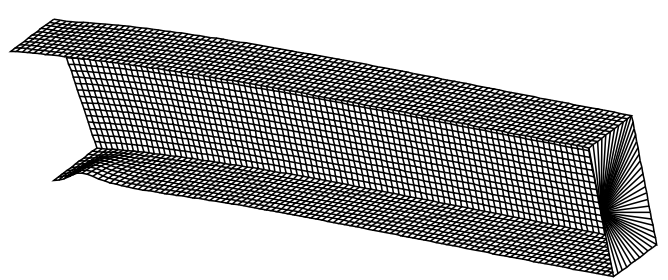

(c) Deformed Configuration (ABAQUS)

Figure 11. Analysis of Cantilever Beam under Distributed Load 


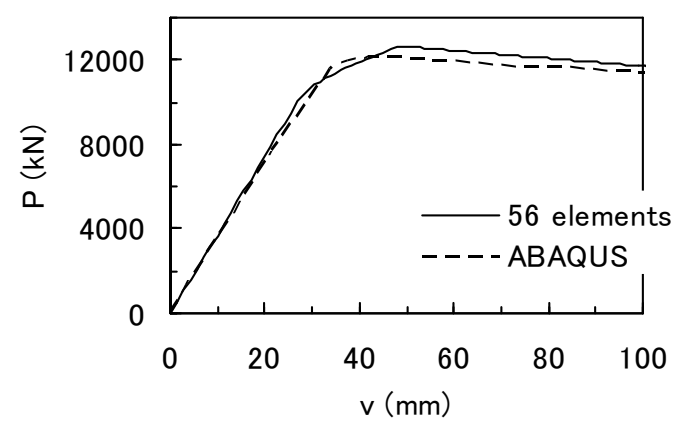

(a) Load $P$ - Displacement $v$ Curves

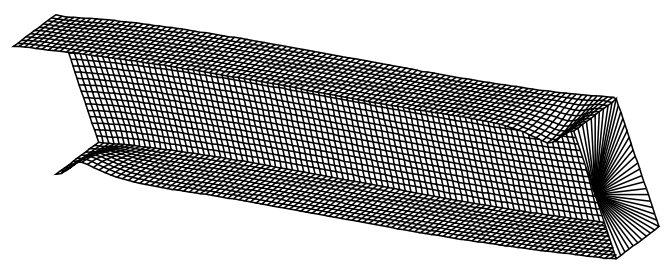

(b) Deformed Configuration (ABAQUS)

Figure 12. Analysis of Fixed-fixed Beam

A half of the fixed-fixed beam (Figure 5(b)) is then analyzed. All the values of the parameters in the constitutive relationship are kept the same as those in the preceding two examples. The numerical result is presented in Figure 12(a) together with that due to ABAQUS. Because of the convergence, only the numerical result by the 56-element mesh is given. The proposed approach agrees well with the ABAQUS result: the difference in the maximum load is $4.20 \%$. Figure 12(b) shows that local buckling occurs in the compression flanges near the fixed end and the middle section of the beam where the concentrated load is applied, which matches the result of the proposed beam-element analysis: LBZs in these two regions undergo the softening behavior. The occurrence of the local buckling in the multiple locations is attributed to the fact that the beam is statically indeterminate. The length of $1400 \mathrm{~mm}$ appears good for both LBZs in the analysis of the fixed-fixed beam.

Based on the results obtained in this section, it may be stated that the trilinear type of constitutive relationship is promising in the practical analysis of a steel beam undergoing local buckling in spite of its extreme simplicity.

\section{CONCLUDING REMARKS}

Local buckling of a steel structure can be simulated well by shell-element analysis. Nevertheless, such an analysis requires much computational cost and is yet to be practical. Hence, effort has been made to implement the structural deterioration due to local buckling in the constitutive relationship so that a beam element can be used for the local buckling analysis of steel structures. However, such a constitutive relationship has a softening branch inevitably, and as demonstrated in this study, simple application of the softening-type constitutive relationship does not lead to mesh objective result.

Against this background, the present study has proposed a finite element procedure that uses the average state variables in the local-buckling zone to control the structural deterioration due to local buckling. The effectiveness of the proposed finite element procedure has been confirmed by solving example problems: the mesh objectivity is shown to be achieved. It is noted that the application of the underlying concept of the proposed procedure is not restricted to the constitutive relationship employed in the present study: it can be applied straightforwardly to any constitutive relationships. 
One of the simplest constitutive relationships that include the influence of the structural deterioration due to local buckling is the one employed in the present numerical examples. At the end, the present study has explored the applicability of this simple trilinear constitutive relationship in comparison with the shell-element analysis by ABAQUS. The results have indicated that this type of constitutive relationship is promising in the practical analysis of a steel beam undergoing local buckling in spite of its extreme simplicity. Yet much remains to be done as to how the bending rigidities and the critical curvature for a given steel member are determined, which is in fact a subject of an on-going project in the author's research group.

\section{REFERENCES}

[1] Public Works Research Institute et al., "Seismic Design for Highway Bridge Piers", Technical Report of Joint Research, PWRI, Ministry of Construction, Japan, 1997.

[2] Goto, Y., Wang, Q. and Obata, M., "FEM Analysis for Hysteretic Behavior of Thin-walled Column", Journal of Structural Engineering, ASCE, 1998, Vol. 124, pp. 1290-1301.

[3] Yamaguchi, E., Nagamatsu, T. and Kubo, Y., "Influence of Finite Element Mesh on Buckling Analysis of Steel Pipe-sectioned Bridge Piers", Fifth World Congress on Computational Mechanics (WCCM V), Paper No. 80566, 2002.

[4] Yamaguchi, E., Abe, K. and Kubo, Y, "Analysis of Steel Bridge Piers Undergoing Local Buckling by Beam Elements", Proceedings of 5th International Colloquium on Stability and Ductility of Steel Structures, 1997, pp. 267-272.

[5] Sakimoto, T., Watanabe, H. and Nakashima, K., "Hysteretic Models of Steel Box Members with Local Buckling Damage", Journal of Structural Mechanics and Earthquake Engineering, JSCE, 2000, No.647/I-51, pp. 343-355.

[6] Watanabe, H. and Sakimoto, T., "Seismic Response Analysis of Concrete-filled Steel Box Piers Considered on Local Buckling", Journal of Structural Mechanics and Earthquake Engineering, JSCE, 2000, No. 647/I-51, pp. 357-368.

[7] Little, G.H., "Rapid Analysis of Plate Collapse by Live Energy Minimisation", International Journal of Mechanical Science, 1974, Vol. 19, No. 12, pp. 725-744.

[8] Hillerborg, A., Modeer, M. and Peterson, P.E., "Analysis of Crack Formation and Crack Growth in Concrete by Means of Fracture Mechanics and Finite Elements", Cement and Concrete Research, 1976, Vol. 6, pp. 773-782.

[9] Bazant, Z.P. and Oh, B.H., "Crack Band Theory for Fracture of Concrete", Materials and Structures, RILEM, 1983, Vol. 16, pp. 155-177.

[10] Yamaguchi, E. and Chen, W.F., "Cracking Model for Finite Element Analysis of Concrete Materials", Journal of Engineering Mechanics, ASCE, 1990, Vol. 116, pp. 1242-1260.

[11] Cook, R.D., Malkus, D.S. and Plesha, M.E., "Concepts and Applications of Finite Element Analysis", 3rd Edition, New York , NY, John Wiley \& Sons, 1989.

[12] Nishino, F. and Hasegawa, A., "Elastic Analysis of Structures", Gihoudo, 1983.

[13] Usami, T. (editor), "Guidelines for Seismic and Damage Control Design of Steel Bridge", Tokyo, Japan, Gihodo, 2006.

[14] Bazant, Z.P., "Nonlocal Damage Theory Based on Micromechanics of Crack Interactions", Journal of Engineering Mechanics, ASCE, 1994, Vol. 120, pp. 593-617.

[15] Khaloo, A.R. and Tariverdilo, S., "Localization Analysis of Reinforced Concrete Members with Softening Behavior", Journal of Structural Engineering, 2002, Vol. 128, pp.1148-1157.

[16] ABAQUS/Standard User's Manual, Ver.5.7, HKS, 1997. 